\title{
Stabilité physique et biologique des milieux interstitiels. Cas de deux stations du Haut-Rhône ${ }^{1}$
}

\author{
Marie-José Dole ${ }^{2}$ \\ Daniel Chessel ${ }^{3}$
}

Mots-Clés : écologie, milieu interstitiel, faune souterraine, fleuve, bras morts, stabilité, effets de perturbation.

Une étude de la stabilité des peuplements interstitiels dans les niveaux supérieurs de la nappe alluviale du Rhône à l'est de Lyon, a été réalisée en relation avec la stabilité physique des milieux et l'action d'une perturbation. Deux stations ont été choisies sur le Haut-Rhóne : l'une est sujette aux remontées de nappe et aux crues de l'Ain (station 8) ; la seconde, alimentée par voie phréatique, ne subit aucune turbulence hydrologique (station 10).

La station la plus exposée à des mouvements hydrologiques présente une biocénose qualitativement et quantitativement très structurée dans un plan vertical, avec une variabilité temporelle négligeable.

La seconde station, plus * calme *, abrite une biocénose moins stratifiée et moins structurèe dans l'espace, caractérisée par une importante variabilité temporelle.

Une modification identique des conditions de milieu :

- ne produit pas de variations notables de la biocénose de la St. 8

- constitue une perturbation à laquelle la faune réagit de manière différente suivant son adaptation au domaine souterrain, dans la St. 10.

Physical and biological stability of interstitial habitats. Case of two stations of the upper Rhone.

Keywords : ecology, interstitial habitats, subterranean fauna, old beds, stability, perturbation effects.

A study of the stability of the interstitial biocenoses in the upper level strata of the alluvial groundwater table of the Rhone river (upstream from Lyon) was undertaken to observe the relation between the physical stability of biotopes and the influence of perturbation. Two interstitial stations were chosen on the upper Rhone : one of the stations is subjected to variations in its groundwater level and to the floods of the Ain river (Station 8); and the second station is only affected by phreatic influences.

Station 8 is most exposed to hydrological variations and has a well-organized biocenose, which has low temporal variability, in its vertical plane.

The other station $(10)$, the most calm, has a less stratified and less spacially-structured biocenoses, with a high temporal variability.

Identical changes in biotope conditions:

- do not produce notable variations in the biocenoses at station 8

- constitute a perturbation to which fauna at station 10 react differently, according to their adaptations to the subterranean biotope.

1. Programme interdisciplinaire de recherche sur l'environnement (PIREN) du CNRS: " Recherches méthodologiques appliquées a la gestion des systèmes fluviaux * dirigée par A.L. Roux.

2. Université Claude Bernard Lyon 1. Département de Biologie Animale et Ecologic. Laboratoire d'Hydrubiologie et Ecologie Souterraines. U.A.CNRS 367, 43, Bd du 11 Novembre 1918,69622 Villeurbanne cédex, France.

3. Université Claude Bernard Lyon 1. Département de Biologie Générale et Appliq̨uée. Laboratoire de Biométrie. U.A. CNRS 243, Biologie des populations, 43, Bd du 11 Novembre 1918,69622 Villeurbanne cédex, France.

\section{Introduction}

L'analyse fonctionnelle d'un hydrosystème complexe comme le Haut-Rhône, a nécessité la prise en compte et l'étude du domaine aquatique souterrain dont il est en partie dépendant. Le rôle du milieu interstitiel se situe essentiellement aux niveaux hydrologique et énergétique : échanges et transferts d'eau, de matière organique et de faune qui contribuent au fonctionnement et à la dynamique du système en milieu non colmaté. 
L'action directe de la faune interstitielle sur la dynamique et sur l'évolution de l'hydrosystème est actuellement moins connue. Danielopol (1983) en fournit un exemple en évoquant le phénomène de "pelletization " 1 , qui préserve la libre circulation de l'eau et de la matière organique. Ce phénomène, important dans " l'anti-clogging process " ralentit le colmatage du sédiment qui est à la base des processus d'évolution et de vieillissement des milieux aquatiques épigés. Dans certains biotopes, il permet le maintien de la porosité du sédiment par la seule action de la faune (Danielopol 1983).

On conçoit donc, à partir de cet exemple, l'intérêt que peuvent présenter la persistance et la stabilité des peuplements interstitiels dans certaines unités fonctionnelles de l'hydrosystème. Les mécanismes qui régissent cette stabilité sont très complexes. Un des premiers facteurs qui permet son maintien est la stabilité physique du milieu. Les questionś qui se posent alors sont relatives à la fois au milieu et à la faune: dans quelle mesure le remaniement continu des sédiments permet-il l'établissement de peuplements interstitiels? Engendre-t-il toujours une instabilité biologique ? Quelles sont les unités systématiques concernées? A l'inverse, qu'observet-on dans une structure physique plus calme?

Ces problèmes ont été abordés au cours de l'étude de deux stations phréatiques du Haut-Rhône.

Dans un précédent article, la structure verticale des peuplements interstitiels des niveaux superieurs de la nappe alluviale du Rhône (" pellicule biologique "), avait été analysée dans trois sites représentat ifs de l'évolution chronologique du fleuve (Dole, 1985). Le présent travail utilise en partie les données faunistiques obtenues au cours de cette étude antérieure.

Elles sont traitées de façon différente dans la mesure où les résultats n'avaient été présentés que sous forme de moyennes, et que l'aspect temporel des variations, donc les caractéristiques dynamiques de la pellicule biologique, n'avaient pas été prises en compte.

1. Méio el macro faune ingèrent les particules fines du sédiment qu'elles rejet tent sous forme de pelotes fécales plus grossières, ce qui modifie la structure du substrat (augmentation de la taille des particules donc des interstices et de la porosité).

\section{1. - Stations - Matériel et méthodes}

Les stations concernées sont les stations 8 et 10 (Gibert \& al. 1977, Dole 1983 b). Leur situation topographique, leur mode d'alimentation hydrogéologique, les particularités physico-chimiques de leur eau interstitielle et la composition biocenotique globale de leurs peuplements sont détaillés dans Dole (1983 b) (fig. 1).

La station 8 appartient à un bras mort encore en contact, à l'aval, avec le chenal vif du fleuve. Située à proximité de la confluence Ain-Rhône, elle est alimentée à la fois par voie souteraine et superficielle. Elle subit en effet les crues de l'Ain, rivière à régime karstique dominant. Le milieu interstitiel correspondant est donc, au moins dans les zones proches de la surface du sédiment, régulièrement modifié par les crues et les remontées du niveau de la nappe alluviale (Stade d'évolution intermédiaire, fig. 1).

La station 10 est située au bord d'une lône isolée du système superficiel actif. Elle dépend uniquement. du lent et régulier apport de la nappe phréatique de la Dombes. Durant la période d'étude (environ 3 ans), cette station n'a jamais subi d'exondation ni de crues. Les variations du niveau d'ont pas excédé, à l'exutoire de la lóne, une trentaine de $\mathrm{cm}$. Contrairement au précédent, ce biotope interstitiel, à l'abri des perturbations exogènes, peut donc être considéré comme une structure physique relativement stable (stade d'évolution ancien, fig. 1).

Suivant une périodicité variable (un mois et demi à deux mois), des prélèvements de faune ont été effectués dans ces deux stations par pompages manuels à la pompe Bou-Rouch (Bou et Rouch 1967; Bou 1974). Les échantillonnages ont été effectués verticalement tous les $50 \mathrm{~cm}$ depuis $-0,5 \mathrm{~m}$ jusqu'à $-1,5$ $\mathrm{m}$ pour la station 8 , et de $-0,5 \mathrm{~m}$ à $-1,5 \mathrm{~m}$ ou $3 \mathrm{~m}$ pour la station 10 . Un volume de 60 litres est aspiré à chaque niveat.

Au plan méthodologique nous soulignerons les choix faits quant à la présentation des résultats et le rôle des méthodes d'analyse multivariées. Analyse en composantes principales et analyse factorielle des correspondances, après avoir fait l'objet d'une mul. titude de descriptions souvent redondantes, appartiennent maintenant à l'appareillage technique de base en écologie. Outil de description des structures multivariées, elles n'en prouvent jamais l'existence mais permettent de choisir le mode de réexpression 


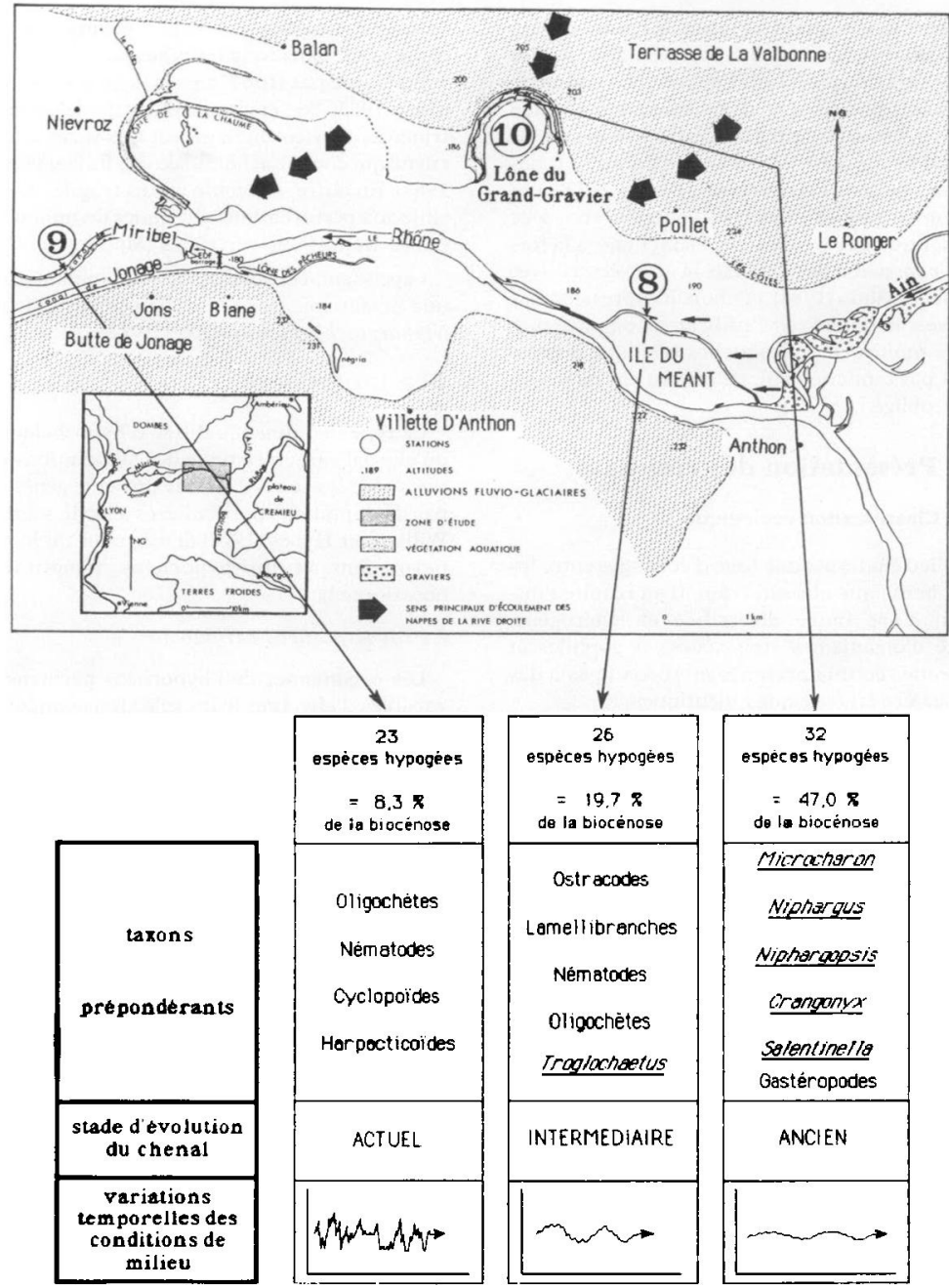

Fig. 1. Situation de la zone d'étude, localisation des stations et rappel des principales caractéristiques biocénotiques de chacune d'elles, d'après Dole 1983 (les taxons soulignés sont des unités systématiques hypogées).

Les variations temporelles des conditions de milieu ont été représentées pour chaque station. La station 9 qui subit le stress hydrologique le plus important a fait l'objet d'une étude particulière en 1984. Les résultats en seront publiés ultérieurement. 
des données qui intègrent au mieux la nature et l'origine de leurs structures internes. En ce sens, les méthodes graphiques, si elles ne sont pas une alternative aux méthodes d'analyse des données (Auda 1983), peuvent redevenir les modes privilégiés de l'expression des résultats expérimentaux et des variations qui ont été retenues (Bertin 1973). Les outils informatiques modernes contribueront à ce choix tactique. Les figures 3 et 5 sont donc, à la fois forme d'expression des résultats bruts exécutée avec le logiciel de Auda (1984), et choix d'expression des structures, impliqué par l'utilisateur de plusieurs analyses multivariées, lesquelles bien que n'apparaissant pas explicitement, ne restent pas moins un passage obligé.

\section{2. - Présentation des résultats}

\section{1. - Classification écologique}

Le milieu étudié est une zone d'échanges entre les milieux benthique et souterrain. Il en résulte l'installation d'une faune diversifiée et hétérogène, mélange d'organismes stygoxènes, stygophiles et stygobiontes dont la présence ne répond pas à des exigences éco-éthologiques identiques.

\subsubsection{FAUNE SOUTERRAINE (STYGOBIONTES)}

\section{(A) (fig. 3 et 5)}

On distingue tout d'abord les formes phréatobies assez strictement liées au milieu interstitiel, dont le genre Microcharon est un exemple typique: la suppression des grains de sable environnants entraîne la mort de l'organisme (Coineau 1971). Ces animaux présentent de nombreuses adaptations morphologiques (dépigmentation, anophtalmie, allongement d'appendices, forme du corps, musculature...), physiologiques, comportementales et thigmotactiques. Ils ne semblent vivre que dans des milieux phréatiques vrais (écoulements lents et réguliers d'une nappe souterraine), calmes et stables, car ce sont souvent des organismes très fragiles.

Exemples : Microcharon, Siettitia, Bathynella, Troglochaetus et, dans une moindre mesure, Salentinella et Proasellus.

Le groupe des Amphipodes troglobies (genres Niphargus, Niphargopsis et Crangonyx) présente également d'importantes adaptations au milieu souterrain. Ces genres ont cependant une plus grande valence écologique que les précédents. "Niphargus est présent dans tous les types de gisements d'eau souterraine pas trop éloignés de la surface du sol » (Ginet 1983). Son environnement spatial, granulométrique et physico-chimique est très varié. C'est l'obscurité qui constitue l'unité de son milkeu vital (Ginet 1983). En outre, il semble moins fragile, moins sensible aux perturbations physiques du milieu et capable de migrations verticales (Mathieu et al. 1985).

Cependant, certaines espèces semblent présenter une préférence pour le milieu phréatique (cas de Niphargus kochianus, Ginet 1982).

\subsubsection{L'HYPORHÉOS}

Ce type de faune, qualifiée d'hyporhéique parce qu'elle est caractéristique des associations rencontrées sous les cours d'eau, ne présente généralement pas d'adaptations particulières à la vie souterraine. William et Hynes (1974) distinguent un hyporhéos permanent et un hyporhéos temporaire (ou occasionnel).

\subsubsection{Hyporhéos permanent}

Les organismes de l'hyporhéos permanent sont capables d'effectuer leur cycle vital complet au sein du milieu interstitiel. Ce biotope n'est cependant pas leur seul milieu de vie puisqu'on les rencontre dans les milieux benthiques et planctoniques. Dans cet ensemble systématique hétérogène et polyvalent on constate la présence de quelques espèces hypogées (cf. liste systématique dans Dole 1983 b).

(B) (fig. 3 et 5) groupe de taxons renfermant à la fois des genres et espèces hypogées (stygobiontes et stygophiles) et épigés (phréatoxènes, stygoxènes). Ce sont les Oligochètes, Nématodes, Ostracodes, Cyclopoìdes et Harpacticoïdes. Cet ensemble forme une sorte de "faune de base "qui se rencontre dans la totalité des prélèvements (occurence $=1$ ), les Gastéropodes ont une présence moins régulière (occurence $\neq 1$ ).

(C) (fig. 3 et 5) groupe de taxons ne renfermant (pratiquement) que des espèces épigées. Ce sont les Rotifères, les Tardigrades, les Hydracariens et les Hydres, qui peuvent at teindre les niveaux profonds $(-1,5 \mathrm{~m})$, sans doute en raison de leur taille réduite, mais aussi les Planaires, les Achètes, les Lamellibranches et les Coléoptères qui sont plus superficiels, et les groupes des Cladocères et des Calanoïdes, plutôt adaptés à la vie planctonique. 


\subsubsection{Hyporhéos temporaire}

(D) (fig. 3 et 5) dans ce cas, les animaux sont contraints de quitter le milieu interstitiel pour achever leur cycle vital. La plupart des larves d'insectes entrent dans cette catégorie : Chironomidae, Ceratopogonidae et autres Diptères, Plécoptères, Trichoptères, Ephéméroptères et Sialidae.

Les Plécoptères Leuctridae occupent une place particulière dans ce groupe car ils présentent un plus grand degré d'adaptation au domaine hyporhéique. Berthelemy (1968) insiste sur l'aspect grèle des larves de Leuctra major et sur leur abondance dans le milieu interstitiel profond où se déroule une grande partie de la vie larvaire (Bou 1979).

\subsubsection{CRUSTACES SUPERFICIELS}

(D) (fig. 3 et 5). Ce sont des organismes benthiques qui pénètrent généralement peu en profondeur. C'est la raison pour laquelle on les fait figurer sous la même trame que les organismes de l'hyporhéos temporaire. Gammarus et surtout Asellus en sont des exemples types.

\section{2. - Station 8}

Les données numériques, variables de 0 à 13400 individus par prélèvement, sont regroupées en 14 classes d'abondance. La matrice comporte 30 colonnes (taxons) et 45 lignes (soit 15 prélèvements à chacun des 3 niveaux d'échantillonnage). Une Analyse Factorielle des Correspondances a montré que la principale source de variation de la biocénose est liée à la profondeur. Les autres axes factoriels ne sont pas significatifs. Le facteur temporel est négligeable.

On propose donc une représentation globale de la biocénose, basée sur des données initiales non transformées. Chaque taxon est représenté par son abondance absolue en fonction du temps et de la profondeur; son abondance relative à chaque niveau d'échantillonnage et sa classification écologique précédemment déterminée d'après les affinités qu'il présente avec le milieu interstitiel et souterrain.

L'abondance absolue figure sous forme d'un petit carré dont la surface est proportionnelle a la densité du taxon dans les prélèvements. Une absence est figurée par un point.

La représentation globale de la biocénose qui a été réalisée ne tient compte que des densités D1, D2, D3 observées respectivement à $-1,5 \mathrm{~m},-1 \mathrm{~m}$ et $-0,5$ $\mathrm{m}$ (fig. 2). Chaque colonne correspond en effet à un classement des taxons par rapport à ces densités.

Par exemple, dans le cas de la première colonne, $\mathrm{D} 1 \simeq \mathrm{D} 2 \simeq 0$. On a à faire à des organismes qui ne sont présents qu'au premier niveau d'échantillonnage. Leur fréquence est de $100 \%$ à $-0,5 \mathrm{~m}$. Leur trame claire indique qu'ils sont peu adaptés au milieu interstitiel.

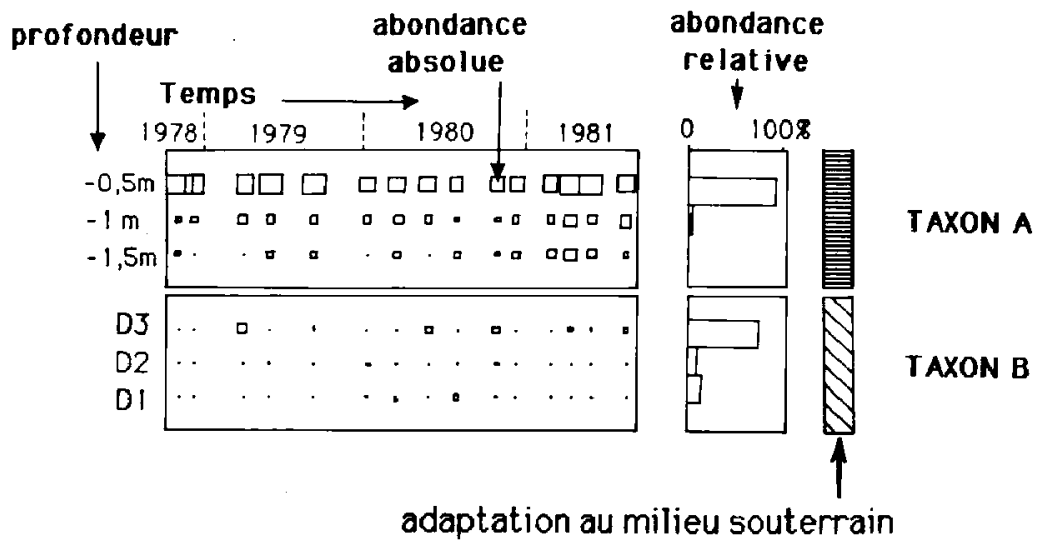

Fig. 2. Station 8 : mode de représentation des taxons (pour la signification exacte des trames, se reporter au texte du $\S$ 2.1 et aux figures 3 ou 5). 


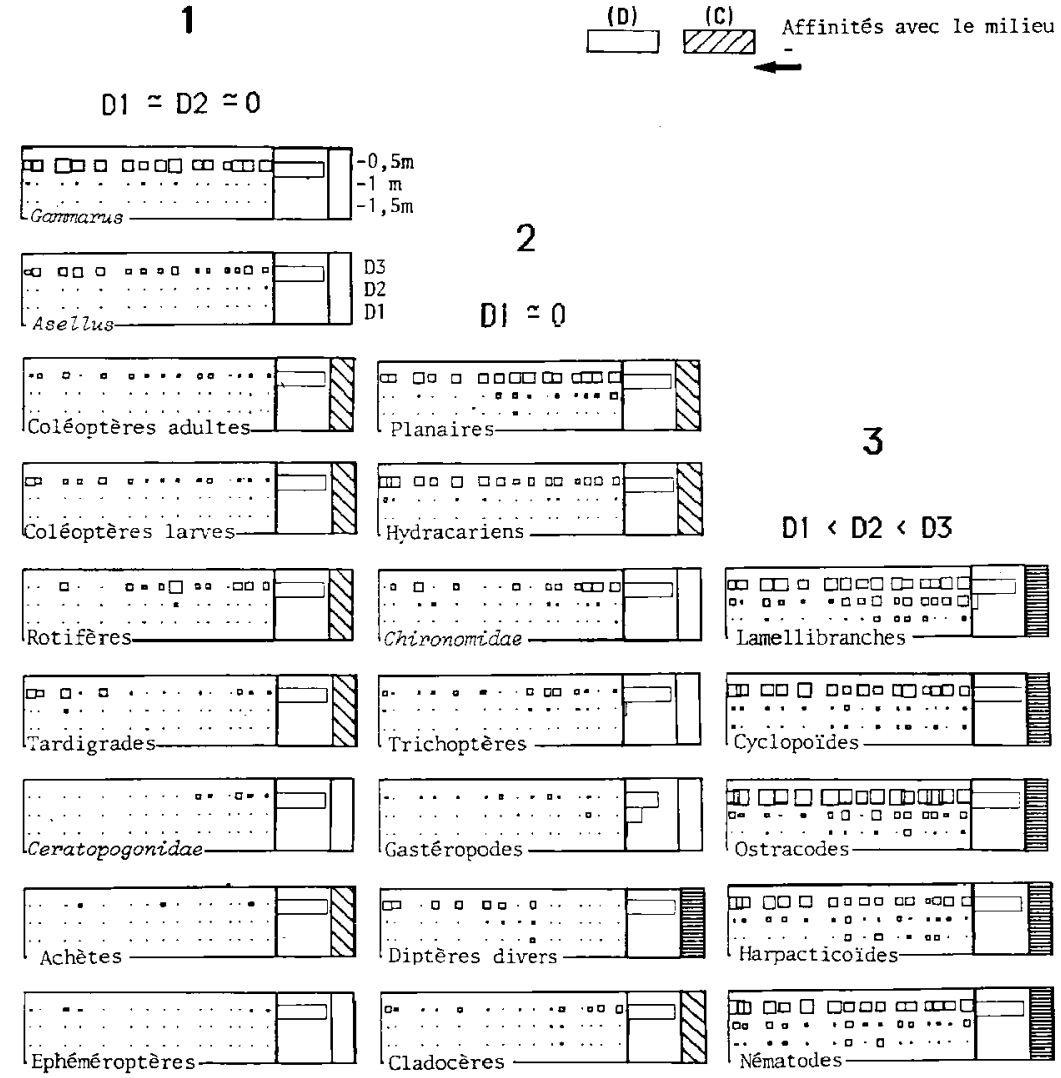

Fig. 3. Représentation globale de la biocénose de la station 8. Le terme * Niphargides * désigne les Amphipodes troglobies appartenant aux genres Niphargus, Niphargopsis et Crangonyx (pour l'explication des let tres (A), (B), (C), (D), et des tra. mes correspondantes, se reporter au texte $\$ 2-1)$. 

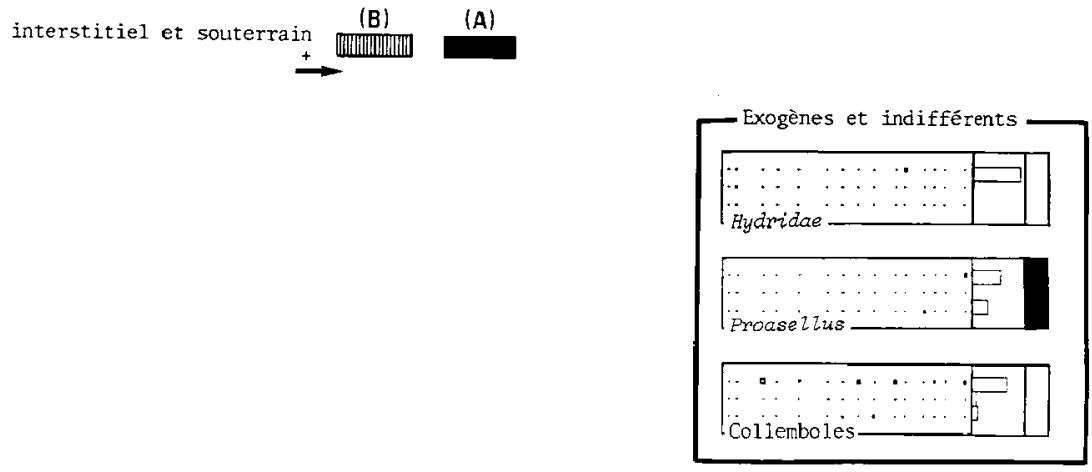

4

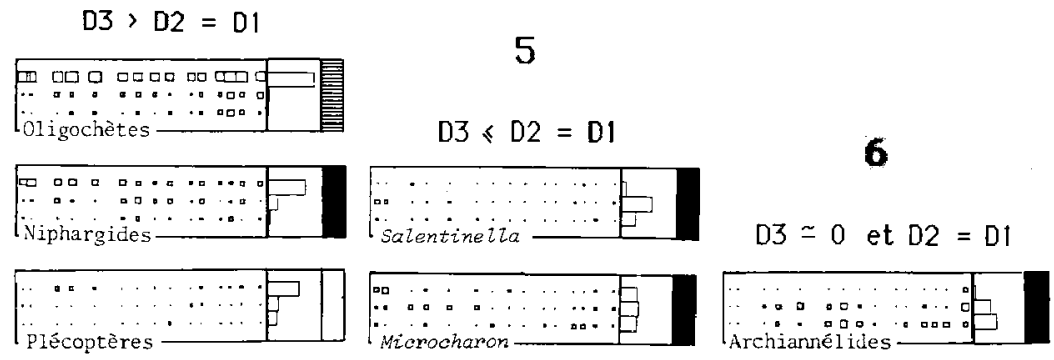


Dans la deuxième colonne, seule D 1 est voisine de zéro (D 2 0 et D 2 0). Les animaux appartenant à cet ensemble pénètrent plus profondément dans le sédiment puisqu'ils commencent à apparầtre au deuxième niveau d'échantillonnage. A l'exception des Gastéropodes, faiblement représentés, il s'agit d'une faune à dominante superficielle.

Dans la troisieme colonne, on observe encore une répartition verticale de type épigé, caractérisée par une diminution régulière de la densité (D $1<\mathrm{D}_{2}$ SD 3). Cependant, les organismes concernés réussissent mieux à exploiter les zones profondes que les précédents (D $1 \neq 0$ ). Ce sont des taxons de l'hyporhéos permanent appelés "faune de base" renfermant également des espèces souterraines (trame foncée).

La composante souterraine augmente dans le quatrième groupe (D 3> D $2=$ D 1) avec l'apparition du taxon stygobie le plus polyvalent : les « Niphargides " (trame noire). Dans cet ensemble, la fréquence des organismes n'est pas toujours de $100 \%$ à $-0,5 \mathrm{~m}$.

Dans le cas extrême (cinquième et sixième colonnes), on constate une inversion de la répartition verticale par rapport aux groupes précédents. La fréquence du genre Troglochaetus est maximale à $1,5 \mathrm{~m}$ et voisine de zéro à $-0,5 \mathrm{~m}$. Les espèces mises en cause sont typiquement phréatobies (trame noire).

Dans cette biocénose, les taxons qui présentent les densités et les occurrences les plus importantes sont rassemblées au niveau du groupe 3, confirmant le fait qu'il s'agit d'une "faune de base ", bien implantée dans le milieu. Ceci s'explique par la composition mixte de ces taxons (espèces épigées + espèces hypogées) qui constitue une adaptation aux deux types de milieux. Les organismes adaptés à la vie souterraine (colonnes 4,5 et 6 ) ou superficielle (colonnes 1 et 2) sont moins abondants. Les peuplements correspondants sont plus diversifiés dans le cas de la faune épigée et le sont moins dans le cas de la faune hypogee.

On est donc en présence d'un modèle relativement. simple et bien organisé, dans lequel les variations temporelles sont peu importantes et où la répartition verticale des organismes, généralement très marquée, varie d'une manière assez logique suivant leur adaptation au milieu interstitiel.

\subsection{Station 10}

\subsubsection{Déroulement de L'Étude}

Le principe de figuration des taxons est le même que celui qui a été utilisé pour la station 8. L'unité systématique est représentée par son abondance en fonction du temps et de la profondeur, et par son degré d'adaptation au milieu souterrain.

Cependant, le déroulement de l'étude est un peu plus complexe ; il s'effectue en deux phases (fig. 4) :

- une première phase (fin 1978 à fin 1980), au cours de laquelle, comme précédemment, les 3 premiers niveaux ont été échantillonnés $(-0,5 \mathrm{~m}$; $1 \mathrm{~m}$ et $-1,5 \mathrm{~m}$ )

- une seconde phase (1981), réalisée après un premier essai effectué en 1980 , et qui comporte 3 niveaux supplémentaires ( $-2 \mathrm{~m},-2,5 \mathrm{~m}$ et $-3 \mathrm{~m}$ ).

Au cours de la seconde période d'étude, une perturbation physique due à la technique d'échantillonnage, est intervenue dans les premiers niveaux de prélèvement. Elle est matérialisée par une flèche verticale. L'introduction de cette perturbation d'origine exogène permettra de tester la réaction des différents taxons aux modifications des conditions de milieu.

L'existence d'une variabilité temporelle non négligeable n'a pas permis un traitement aussi simple que dans le cas de la station 8 . Pour classer la faune et modéliser la biocénose, on a du recourir à une Analyse en Composantes Principales. Le résultat de ce traitement fait l'objet de la figure 5.

Cornme précédemment, on constate l'existence de taxons superficiels qui ne sont jamais présents dans les niveaux inférieurs (Gammarus, Lamellibranches et « superficiels rares") et d'un phréatobie (Bathynella) qui, au contraire, n'est rencontré qu'en dessous de $1,5 \mathrm{~m}$ de profondeur.

- En dehors de ces cas particuliers, pendant la période de fonctionnement "normal " du système (première phase de l"étude), la stratification des organismes est beaucoup moins nette que dans la station 8 ; elle est souvent variable au cours du temps (interaction espace-temps). Il semble qu'il existe une interférence entre les niveaux, ou un mélange spation-temporel de la faune. Ce mélange est beaucoup plus important que dans la station 8 qui présente une organisation spatiale beaucoup plus précise et constante. Cette répartition verticale est 


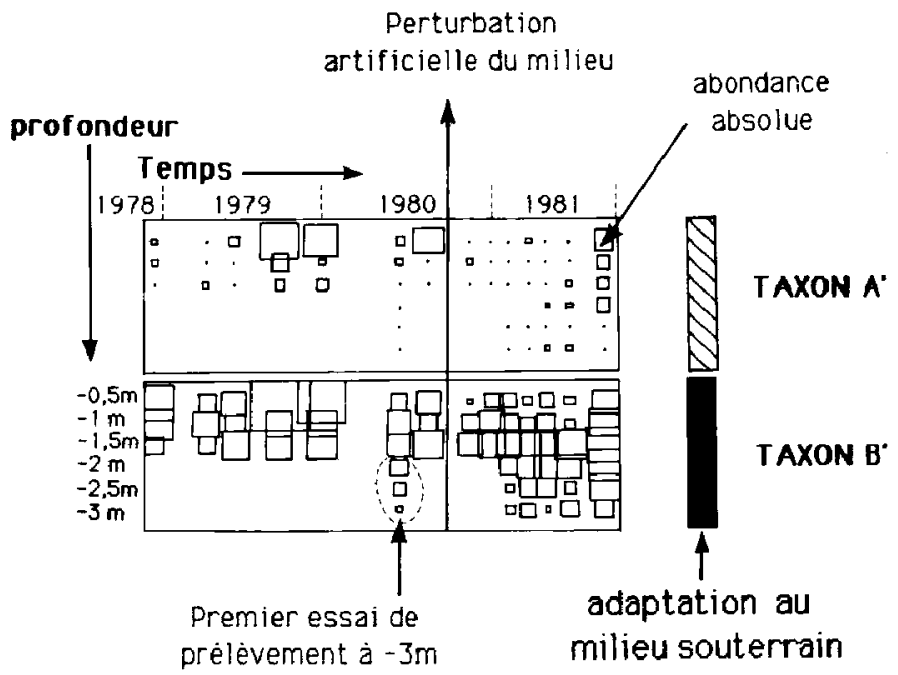

Fig. 4. Station 10 : mode de représentation des taxons (pour la signification exacte des trames, se reporter au texte du $\S 2-1$ et aux figures 3 ou 5 ).

généralement caractérisée par une diminution de densité des organismes avec la profondeur, quelles que soient les affinités qu'ils présentent avec le milieu souterrain : les groupes stygobiontes ne montrent pas la strafication qui leur est pourtant caractéristique. On peut citer l'exemple de Troglochaetus qui présente, dans la station 8 , la répartition verticale de type hypogée la plus marquée et qui, ici est souvent plus abondant à proximité de la surface.

- Au cours de la seconde phase d'étude, la perturbation du milieu permet de distinguer deux types de réactions :

* une réaction de disparition des organismes, qui est d'autant plus nette que les abondances ont été fortes au cours de la première phase de l'étude. Ce sont surtout des organismes à tendance superficielle (trames claires) qui présentent ce type de réaction. Tout se passe comme si cette faune quittait, activement ou passivement, le milieu devenu inhospitalier.

* une réaction d'enfouissement de la faune, qui cher. che refuge dans les zones profondes. Ce sont les taxons les mieux adaptés au domaine souterain qui montrent ce second type de réaction. Pour certains, l'enfouissement se prolonge (Niphargides, Salentinelles). Pour d'autres, on observe un début de retour à l'état antérieur, vers la fin de l'étude.

Sur le modèle ( $\mathrm{fig} .5$ ), la situation observée est schématisée, pour chaque taxon, par une flèche horizontale, droite dans le cas de la réaction de disparition, courbe dans le cas de la réaction d'enfouissement. Les zones sombres à l'in térieur de la flèche corres. pondent aux périodes d'abondance des animaux.

\section{3. - Discussion}

La notion de stabilité peut être perçue à partir du concept de perturbation. La composante statique de la stabilité désigne les propriétés de constance ou de persistance des écosystèmes, éventuellement liées à la permanence des conditions environnementales (Barbault 1983). Or, à l'échelle de temps considérée, chacune des deux stations est bien 


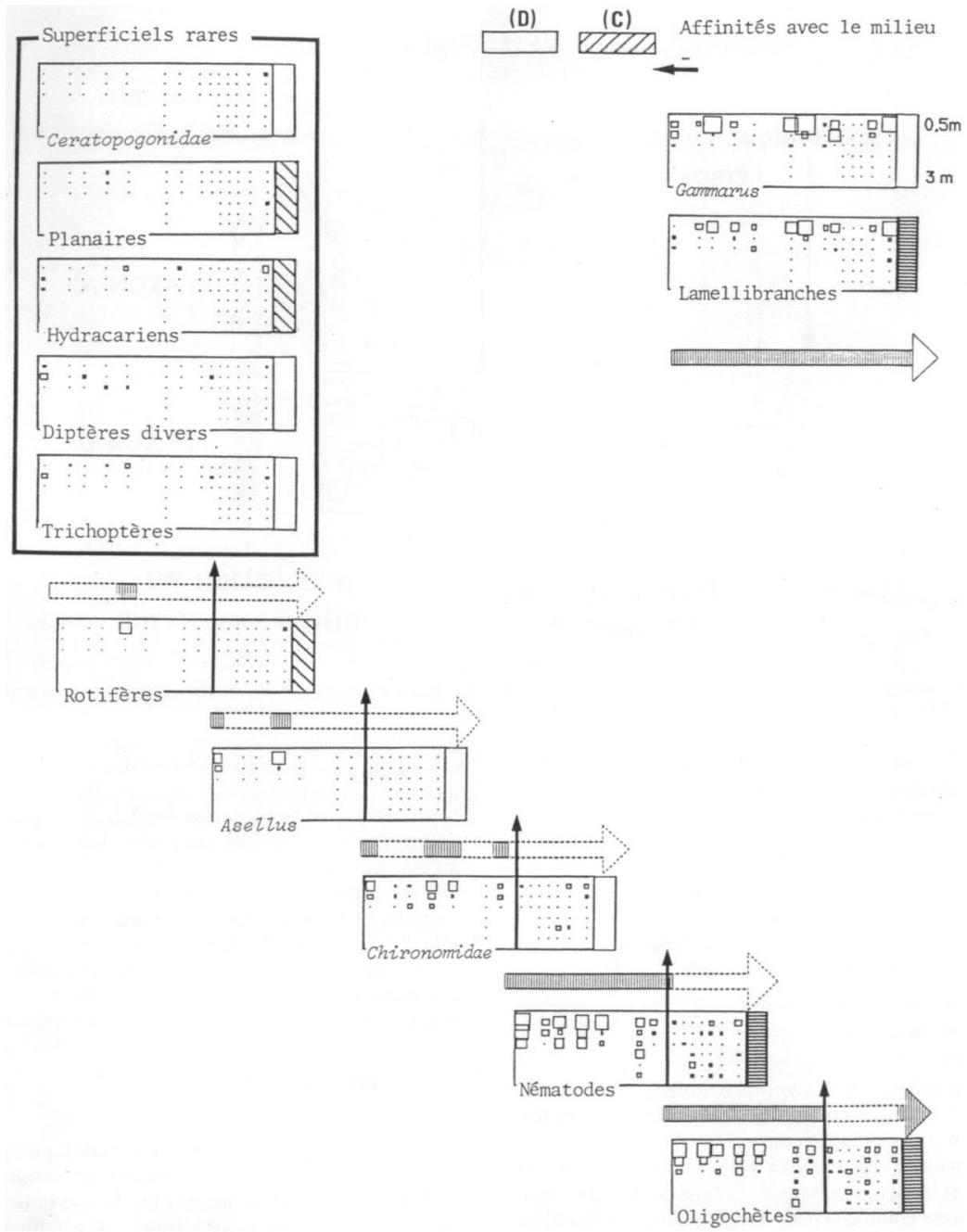

Fig. 5. Représentation globale de la biocénose de la station 10. Le terme a Niphargides " désigne les Amphipodes troglo. bies appartenant aux genres Niphargus, Niphargopsis et Crangonyx (pour l'explication des lettres (A), (B), (C), (D) et des trames correspondantes, se reporter au texte du $\$ 2-1$ ). 


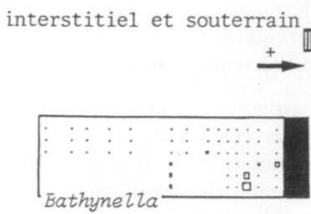

(B)

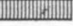

(A)

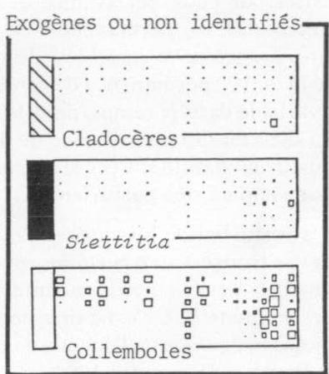

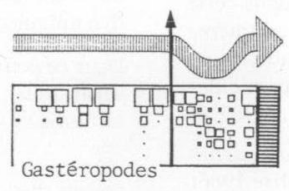
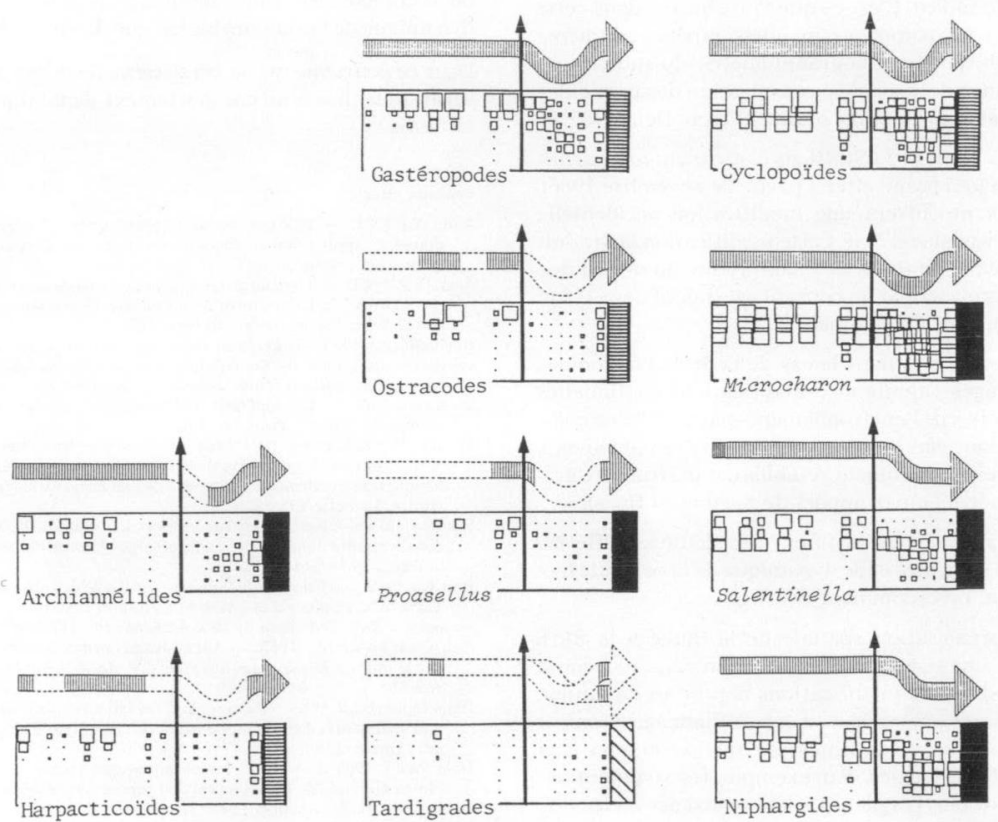
caractérisée par cette persistance des conditions environnementales: permanence de conditions constantes (ou très peu variables) du milieu, dans le cas de la St. 10 ; permanence de conditions d'armplitude variable dans le temps, dans le cas de la St. 8. On est donc en fait en présence de deux aspects différents de la stabilité. Ceci est a contrario confirmé par l'impact des perturbations.

"Il y a perturbation lorsque telle ou telle variable d'état se trouve à une certaine époque hors de la gamme de valeurs correspondantes sous l'effet de causes accidentelles, c'est-à-dire non répétitives ou du moins irrégulières à l'échelle de temps considérée " (Blandin \& Lamotte 1984).

L'action d'un même phénomène peut donc se traduire par une perturbation, ou une absence de perturbation, suivant la succession des événements dans le milieu. C'est ce que l'on observe dans cette étude: les pompages manuels répétés au même endroit modifient la granulométrie du substrat au voisinage de la sonde par aspiration des particules fines et sableuses du sédiment (voir Dole 1983 a).

* Dans le cas de la St. 10, ils constituent une pertur. bation (qui prend effet à partir de novembre 1980), car ils produisent une modification accidentelle d'une variable d'état. Cette modification se traduit par une diminution de l'habitabilité du milieu due à l'instauration d'un courant ascendant de $-0,5 \mathrm{~m}$ à la surface du sédiment.

* Au contraire, dans le cas de la St. 8, l'action des pompages s'ajoute aux modifications continuelles naturelles de l'environnement spatial. Elle ne constitue donc plus une perturbation (les remaniements réguliers du sédiment rétablissent la structure granulométrique par apport de particules fines).

Ces mécanismes justifient sans doute les différen. ces de structure et de dynamique de la pellicule biologique précédemment décrites :

- L'organisation spatiale de la faune à la St. 8 serait une stratégie de répartition de la biocénose répondant aux modifications régulières du milieu. A chaque profondeur d'èchantillonnage seraient associés les organismes les mieux adaptés aux conditions locales. Par exemple, les stygobiontes, souvent plus fragiles que les stygoxènes ou les ubi. quistes sont aussi les seuls capables d'exploiter les zones profondes plus calmes. La persistance de la biocénose serait donc ici liée à la structure spatio- temporelle de la biocénose, c'est-à-dire, à la stratification qualitative et quantitative et à la constance de la faune (variabilité temporelle négligeable).

- Dans la St. 10, au cours de la première phase d'étude, on observe le phénomène inverse, c'est-àdire une inorganisation spatiale et une variabilité temporelle non négligeable des peuplements. Il semble que la biocénose, plus riche en phréatobies, soit plus "plastique " et répartie de manière moins stricte que dans la St. 8, sans doute parce que les conditions dynarniques du milieu sont moins restrictives vis à vis de ces organismes. C'est seulement l'action de la perturbation exogène qui produit une organisation de la faune traduite par les deux types de réaction: disparition ou enfouissement. La remontée ul térieure des organismes vers la surface du sédiment évoque les phénomènes de résilience ou d'homéostasie qui constituent la composante dynamique de la stabilité biologique (Barbault 1983).

Dans ce deuxième cas, la persistance de la biocénose serait donc liée à un comportement dynamique de la faune.

\section{Travaux cités}

Auda (Y.). 1983. - Róle des méthodes graphiques en analyse des données : application au dépouillement des enquêtes écologiques. Thèse Zème cycle, Univ. Lyon : $127 \mathrm{p}$.

Auda (Y.7. 1984. - Logiciel graphique pour l'anal yse des données (FORTRAN 77). Laboratoire de Biométrie, Université Lyon 1, 69622 Villeurbanne cédex, Ronéo: $107 \mathrm{p}$

Barbault (R.). 1983. - Écologie Générale. Masson, Paris : 224 p.

Berthélémy (C.). 1968. - Contribution à la connaissance des Leuctridae (Plecoptères). Annls Limnol., 4 (2): 175-198.

Bertin (J.). 1973. - La grophique et le trattement graphique de l'information. Villars, Paris : $431 \mathrm{p}$.

Blandin (P.) \& Lamotte (M.). 1984. - Ecologie des systèmes et aménagement : fondements théoriques et principes méthodo. logiques. In Fondernents rationnels de l'aménagement d'un territoire. Lamotte ED. : 270.291.

Bou (C.). 1974. - Recherches sur les eaux souteraines. 25. Méthodes de récolte dans les eaux souterraines interstitielles. Ann. Spéléol., $29(4): 61)-619$.

Bou (C.). 1979. - Etude de la faune interstitielle des alluvions du Tarn. Mise en place d'une station d'érude et résul tats préliminaires. Bull. Féd. Tarn Spéléo. Archéol., 16:117-119.

Bou (C.) \& Rouch (R.) . 1967. - Un nouveau champ de recherches sur la faune aquatique souterraine. C.R. Acad. Sci. Paris, 265 : 369-370.

Danielopol (D.L.). 1983. - Ecological investigations on the alluvial sediments of the Danube in the Vienna area. Verh. internat. Verein. Limtol. $22 ; 1755-1761$.

Dole (M.J.). 1983 a. - Le domaine aquatique souterrain de la plaine alluviale du Rhône à l'est de Lyon : écologie des niveaux supérieurs de la nappe. These 3ème cycle, Univ. Lyon : $168 \mathrm{p}$.

Dole (M.J.). 1983 b. - Le domaine aquatique souterrain de la plaine alluviale du Rhône et l'est de Lyon. 1. Diversité hydrologique et biocénotique de trois stations représentatives de la dynamique fluviale. Vie et Milieu, 33 (3/4) : 219-229. 
Dole (M.J.). 1985. - Le domaine aquatique souterain de la plaine alluviale du Rhône à l'est de Lyon. 2 . Structure verticale des peuplements des niveaux superieurs de la nappe. Stygologia 1 (3) $270-291$.

Gibert (J.), Ginet (R.), Mathieu (J.) \& Reygrobellet (J.L.). 1977. Structure et fonctionnement des écosystemes du Haut Rhòne français. IV. Le peuplement des eaux phréatiques. Premiers résultats. Annls Limnol. 13 (1): 83-97.

Ginet (R.). 1982. - Structure et fonctionnement des écosystèmes du Haut Rhône français. XXIV. Les Amphipodes des eaux interstielles en amont de Lyon. Pol. Arch. Hydrobiol., 29 : 231-237.
Ginet (R.). 1983. - Les Niphargus (Amphipodes souterrains) de la région de Lyon (France). Observations biogéographiques, systématiques et écologiques. Mém. Btospéléol, X : 179-187.

Mathieu (J.), Martin (M.) \& Huissoud (P.) 1984. - Influence des conditions hydrologiques sur l'évolution de la structure et de la démographie d'une population phréatique de l'Amphipode Niphargus rhenorhodanensis. Premiers résultats. Mém. Biospeleol. XI ; 27-36.

Williams (D. D.) \& Hynes (H.B.N.) 1974. - The accurence of ben. thos deep in the substratum of a stream. Freshwat. Biol. 4 : 233-256. 